\title{
Comparison and Prognostic Validation of Multiple Methods of Quantification of Myocardial Blood Flow with ${ }^{82}$ Rb PET
}

\author{
Venkatesh L. Murthy ${ }^{1,2}$, Benjamin C. Lee ${ }^{3}$, Arkadiusz Sitek ${ }^{4}$, Masanao Naya ${ }^{1}$, Jonathan Moody ${ }^{3}$, Vivek Polavarapu ${ }^{2}$, \\ Edward P. Ficaro ${ }^{2,3}$, and Marcelo F. Di Carli ${ }^{1}$ \\ ${ }^{I}$ Noninvasive Cardiovascular Imaging Program, Departments of Radiology and Medicine, Brigham and Women's Hospital, Harvard \\ Medical School, Boston, Massachusetts; ${ }^{2}$ Division of Nuclear Medicine, Department of Radiology, University of Michigan, Ann \\ Arbor, Michigan; ${ }^{3}$ INVIA Medical Imaging Solutions, Ann Arbor, Michigan; and ${ }^{4}$ Division of Nuclear Medicine, Department of \\ Radiology, Massachusetts General Hospital, Boston, Massachusetts
}

The quantification of myocardial blood flow (MBF) and myocardial flow reserve (MFR) using PET with ${ }^{82} \mathrm{Rb}$ in patients with known or suspected coronary artery disease has been demonstrated to have substantial prognostic and diagnostic value. However, multiple methods for estimation of an image-derived input function and several models for the nonlinear first-pass extraction of ${ }^{82} \mathrm{Rb}$ by myocardium have been used. We sought to compare the differences in these methods and models and their impact on prognostic assessment in a large clinical dataset. Methods: Consecutive patients $(n=2,783)$ underwent clinically indicated rest-stress myocardial perfusion PET with ${ }^{82} \mathrm{Rb}$. The input function was derived using a region of interest (ROI) semiautomatically placed in the region of the mitral valve, factor analysis, and a hybrid method that creates an $\mathrm{ROI}$ from factor analysis. We used 5 commonly used extraction models for ${ }^{82} \mathrm{Rb}$ to estimate MBF and MFR. Pearson correlations, bias, and Cohen $\mathrm{k}$ were computed for the various measures. The relationship between MFR/stress MBF and annual rate of cardiac mortality was estimated with spline fits using Poisson regression. Finally, incremental value was assessed with the net reclassification improvement using Cox proportional hazards regression. Results: Correlations between MFR or stress MBF measures made with the same input function derivation method were generally high, regardless of extraction model used (Pearson $r>0.90$ ). However, correlations between measures derived with the ROI method and other methods were only moderate (Pearson $r=0.42-0.62$ ). Importantly, substantial biases were seen for most combinations. We saw that the relationship between cardiac mortality and stress MBF was variable depending on the input function method and extraction model, whereas the relationship between MFR and risk was highly consistent. Net reclassification improvement was comparable for most methods and models for MFR but was highly variable for stress MBF. Conclusion: Although both stress MBF and MFR can improve prognostic assessment, MFR is substantially more consistent, regardless of choice of input function derivation method and extraction model used.

Key Words: myocardial perfusion imaging; myocardial flow reserve; ${ }^{82} \mathrm{Rb}$

J Nucl Med 2014; 55:1952-1958

DOI: 10.2967/jnumed.114.145342

Received Jul. 5, 2014; revision accepted Oct. 8, 2014.

For correspondence or reprints contact: Venkatesh Murthy, University of Michigan, 1500 E. Medical Center Dr., SPC 5873, Ann Arbor, MI 48109-5873. E-mail: vlmurthy@med.umich.edu

Published online Nov. 26, 2014.

COPYRIGHT (C 2014 by the Society of Nuclear Medicine and Molecular Imaging, Inc.
$\mathbf{N}$ oninvasive quantification of myocardial blood flow (MBF) and myocardial flow reserve (MFR) with PET has been shown in several clinical cohorts to improve prognostic (1-6) and diagnostic (7-10) assessment of coronary artery disease (CAD). However, at least 5 models (Fig. 1) are commonly used for the correction of nonlinear extraction of ${ }^{82} \mathrm{Rb}$ when quantifying MBF (11-16). Furthermore, the arterial blood pool input function has been traditionally determined using region-of-interest (ROI) methods, although factor analysis methods have been developed more recently and may have important advantages in reproducibility and accuracy $(17,18)$. Substantial methodologic variation exists in the literature. Small studies have suggested that, on average, different software tools generate similar estimates for MFR, the ratio of stress to rest MBF (19-21), potentially due to cancellation of systematic differences. Further, these studies suggest that although population average MFR estimates are similar and correlations between different methods are reasonable, a substantial variation in individual measurements exists. No prior study comparing different methodologies has used a gold standard based on anatomy or clinical outcomes.

We sought to systematically compare the effects of 3 methods for estimation of the arterial input function and 5 validated extraction models for ${ }^{82} \mathrm{Rb}$ on the incremental prognostic value of blood flow quantification with PET in patients with known or suspected CAD.

\section{MATERIALS AND METHODS}

\section{Study Population}

We evaluated a previously described cohort of all patients referred for rest-stress cardiac PET at the Brigham and Women's Hospital between January 1, 2006, and June 30, 2010 (3). The Partners Healthcare Institutional Review Board approved this retrospective study, and the requirement to obtain informed consent was waived.

\section{PET Imaging}

Patients were studied with a whole-body PET/CT scanner (Discovery RX or STE LightSpeed 64; GE Healthcare) in 2-dimensional mode using $1,480-2,200 \mathrm{MBq}$ of ${ }^{82} \mathrm{Rb}$ as a flow tracer at rest and stress as has previously been described (3). Dynamic datasets were reconstructed using ordered-subsets expectation maximization with an all-pass filter into 27 frames $(14 \times 5,6 \times 10,3 \times 20,3 \times 30$, and $1 \times 150 \mathrm{~s})$. Maximal coronary vasodilation was achieved using dipyridamole, adenosine, regadenoson, or dobutamine, as clinically appropriate. PET images were evaluated semiquantitatively to quantify clinically overt myocardial scarring and ischemia (22) using the Corridor4DM software (INVIA Medical Imaging Solutions). 


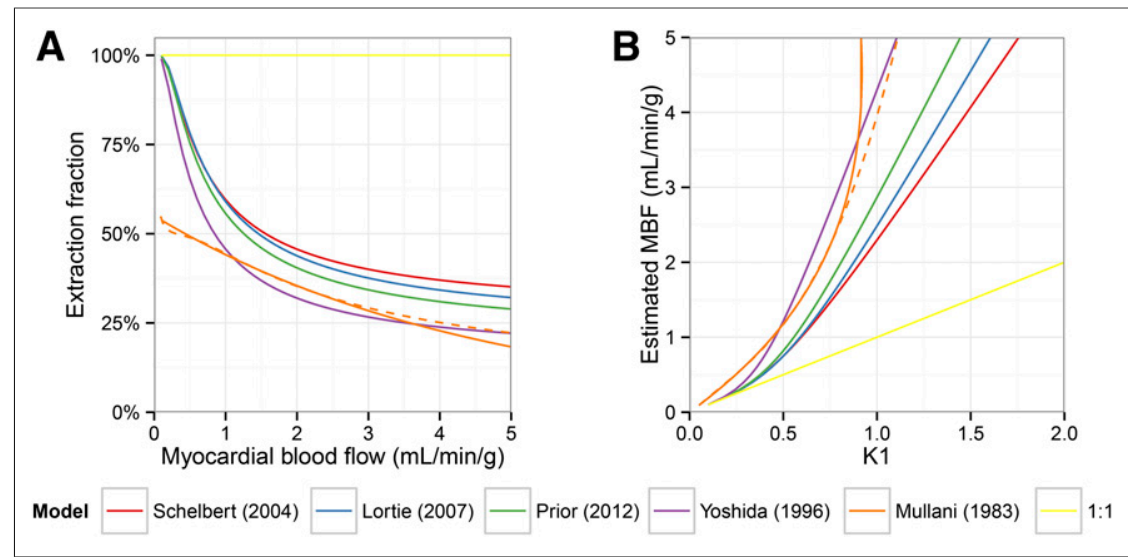

FIGURE 1. Variety of kinetic models. (A) All 5 of the commonly used models for extraction of ${ }^{82} \mathrm{Rb}$ into myocardium predict substantial decrease in extraction for blood flows in physiologic range. (B) Consequently, estimated MBF grows more rapidly than $K_{1}$ rate constant, amplifying uncertainty in blood flow estimates at ranges typical of stress hyperemia. Because the Mullani model is not defined for flows above $4.55 \mathrm{~mL} / \mathrm{min} / \mathrm{g}$ and $K_{1}$ above 0.92 , we modified this model slightly to be estimable uniquely across entire range of blood flows and $K_{1}$ values (dashed lines).

death certificates and were adjudicated by 2 cardiologists in a blinded manner. Cause of death could not be accurately adjudicated in 16 of 279 cases.

\section{Statistical Analysis}

Statistical significance was assessed with Wilcoxon tests, Fisher exact tests, and $\chi^{2}$ tests for continuous, dichotomous, and categoric variables, respectively. MFR and stress MBF computed with different methods were compared with paired $t$ tests and Pearson correlation coefficients. Categorized MFR as $<1.5,1.5-2.0$, and $>2.0$ were compared for different techniques using weighted Cohen $\kappa$ (discrepancies were weighted by number of categories difference).

Poisson regression with generalized additive models was used to fit cubic splines for annualized event rates. Cox proportional hazards regression was used to adjust for the effects of clinical risk factors, left ventricular systolic function, and traditional

\section{Flow Quantification}

MBF at rest and stress was quantified using Corridor4DM. For each dataset, the arterial input function was quantified from dynamic images using 1 of 3 methods: a semiautomatically generated 3-dimensional region of interest $(\mathrm{ROI})(6.5 \times 6.5 \times 30 \mathrm{~mm})$ spanning the mitral valve, factor analysis $(17,18)$, or a hybrid method with an ROI comprising the 20 highest-intensity pixels identified by factor analysis. Further details about the ROI method are provided in the supplemental material (available online at http://jnm.snmjournals.org). For the factor analysis and hybrid factor analysis methods, a right ventricular blood-pool time-activity curve was also quantified. The timeactivity curve for the left ventricular myocardial tissue was generated using a semiautomatically generated ROI and was identical regardless of method used to determine the input function. The data were fit to a 2-compartment kinetic model (1-tissue compartment) to obtain 3 sets of estimates for $K_{1}$ and $k_{2}$ for each of the 3 methods. For the factor analysis and hybrid factor analysis methods, spillover from both the right and the left ventricles was also modeled. For the ROI method, only spillover from the left ventricle was modeled. To compute absolute MBF, each of the 3 sets of kinetic rate constants was entered into each of 5 validated extraction models for ${ }^{82} \mathrm{Rb}$ : Mullani et al. (11), Yoshida et al. (13), Schelbert et al. (14), Lortie et al. (15), and Prior et al. (16). Because the model of Mullani et al. (11) has unstable behavior at high flows, several variations have been used. For this study, we used a Taylor series approximation of the original model [extraction fraction $=K_{1} /\left(2.86729 \times K_{1}^{3}-1.11067 \times K_{1}^{2}+2.21469 \times\right.$ $\left.K_{1}-0.01721\right)$ and $\mathrm{MBF}=2.86729 \times K_{1}^{3}-1.11067 \times K_{1}^{2}+2.21469 \times$ $\left.K_{1}-0.01721\right]$, which is shown as a dashed line in Figure 1 . The model of Glatting et al. (12) was not included as it has been supplanted by that of Schelbert et al. (14), which was developed with the same dataset using the Renkin-Crone formalism $(23,24)$. A sixth counterfactual model assuming $100 \%$ extraction of ${ }^{82} \mathrm{Rb}$ was also included (1:1). In total, for each PET examination, 18 different rest and stress MBF estimates were generated. MFR was computed as the ratio of stress-rest MBF for each of these 18 combinations of arterial input function computation method and extraction model.

\section{Assessment of Outcomes}

The primary outcome was death from cardiac causes. Outcomes were ascertained by a combination of public (Social Security Death Index and National Death Index) and institutional databases as well as semiquantitative measures of left ventricular perfusion. The incremental values of MFR and stress MBF were assessed with the continuous net reclassification improvement (NRI) and the c-index. Confidence intervals for these measures were constructed with bootstrap resampling with 1,000 replicates. All statistical analyses were performed with SAS 9.4 (SAS Institute Inc.) or R 3.1.0 (The R Foundation for Statistical Computing).

\section{RESULTS}

The characteristics of patients in this study are summarized in Table 1 and have previously been described in detail (3). During a median follow-up of $1.4 \mathrm{y}$ (interquartile range, 0.7-2.4 y), 137 deaths from cardiac causes occurred.

\section{Variability in Kinetic Parameters}

There is substantial variability in the estimated $K_{1}$ and $k_{2}$ parameters at both rest and stress based on which method of input function estimation is used (Supplemental Fig. 1; Supplemental Table 1). The ROI method resulted in higher estimates for $K_{1}$ than the factor analysis and hybrid methods. Both the ROI and the hybrid methods generated higher estimates for $k_{2}$ than the factor analysis method. Interestingly, when the ROI method was used, the estimates for $K_{1}$ and $k_{2}$ were moderately correlated (Pearson $r=0.41$ and 0.51 for stress and rest, respectively; both $P<$ 0.0001). No meaningful correlations between $K_{1}$ and $k_{2}$ were seen when the hybrid method was used $(r=0.01$ and -0.02 for stress and rest, respectively; $P=0.72$ and 0.23 , respectively). Although statistically significant in this large sample, only minimal correlations were seen between $K_{1}$ and $k_{2}$ with factor analysis $(r=0.08$ and 0.15 for stress and rest, respectively; both $P<0.0001)$.

\section{Variability in Stress MBF and MFR Estimates}

The distributions of stress MBF for each of the 5 extraction models for ${ }^{82} \mathrm{Rb}$ were substantially different when the ROI method was used (Fig. 2; Supplemental Table 2). In contrast, when the hybrid and factor analysis methods were used, the estimated $K_{1}$ fell into a range where the various extraction models were nearly identical. Consequently, the estimated distribution of stress MBF by these input function estimation methods was similar regardless 
TABLE 1

Patient Characteristics

\begin{tabular}{|c|c|c|c|c|}
\hline Variable & $\begin{array}{l}\text { No cardiac death } \\
\quad(n=2,646)\end{array}$ & $\begin{array}{l}\text { Cardiac death } \\
\quad(n=137)\end{array}$ & $\begin{array}{l}\text { All patients } \\
(n=2,783)\end{array}$ & $P$ \\
\hline \multicolumn{5}{|l|}{ Demographic } \\
\hline Age $(y)$ & $64.3[55.8-74.6]$ & 74.6 [63.7-81.4] & $64.8[56.1-75.2]$ & $<0.0001$ \\
\hline Male sex & $1,242(46.9 \%)$ & $91(66.4 \%)$ & 1,333 (47.9\%) & $<0.0001$ \\
\hline Hispanic & $300(11.3 \%)$ & $3(2.2 \%)$ & $303(10.9 \%)$ & 0.0002 \\
\hline Race & & & & 0.06 \\
\hline White & $1,662(62.8 \%)$ & $100(73.0 \%)$ & $1,762(63.3 \%)$ & \\
\hline Black & $436(16.5 \%)$ & $17(12.4 \%)$ & $453(16.3 \%)$ & \\
\hline Other/unknown & $548(20.7 \%)$ & $20(14.6 \%)$ & $568(20.4 \%)$ & \\
\hline \multicolumn{5}{|l|}{ Risk factor } \\
\hline Body mass index $\left(\mathrm{kg} / \mathrm{m}^{2}\right)$ & 29 [25.1-34.7] & $26.4[23-30.4]$ & $28.8[25.1-34.4]$ & $<0.0001$ \\
\hline$\geq 30 \mathrm{~kg} / \mathrm{m}^{2}$ & $1,167(44.1 \%)$ & $37(27.0 \%)$ & $1,204(43.3 \%)$ & $<0.0001$ \\
\hline Hypertension & $2,154(81.4 \%)$ & $117(85.4 \%)$ & $2,271(81.6 \%)$ & 0.26 \\
\hline Dyslipidemia & $1,756(66.4 \%)$ & $97(70.8 \%)$ & $1,853(66.6 \%)$ & 0.31 \\
\hline Diabetes & $940(35.5 \%)$ & $68(49.6 \%)$ & $1,008(36.2 \%)$ & 0.001 \\
\hline Family history of CAD & $723(27.3 \%)$ & $33(24.1 \%)$ & $756(27.2 \%)$ & 0.43 \\
\hline Tobacco use & $276(10.4 \%)$ & $18(13.1 \%)$ & $294(10.6 \%)$ & 0.06 \\
\hline Dialysis & $118(4.5 \%)$ & $15(11.0 \%)$ & $133(4.8 \%)$ & 0.003 \\
\hline \multicolumn{5}{|l|}{ Indication } \\
\hline Chest pain & $1,268(47.9 \%)$ & $38(27.7 \%)$ & $1,306(46.9 \%)$ & $<0.0001$ \\
\hline Dyspnea & 791 (29.9\%) & $61(44.5 \%)$ & $852(30.6 \%)$ & 0.0006 \\
\hline Post-myocardial infarction & $223(8.4 \%)$ & $28(20.4 \%)$ & $251(9.0 \%)$ & $<0.0001$ \\
\hline Preoperative & $380(14.4 \%)$ & $28(20.4 \%)$ & $408(14.7 \%)$ & 0.06 \\
\hline \multicolumn{5}{|l|}{ Cardiovascular history } \\
\hline Any prior CAD & $1,070(40.4 \%)$ & $105(76.6 \%)$ & $1,175(42.2 \%)$ & $<0.0001$ \\
\hline Recent myocardial infarction ( $\leq 30 \mathrm{~d}$ ) & $279(10.5 \%)$ & $37(27.0 \%)$ & $316(11.4 \%)$ & $<0.0001$ \\
\hline Remote myocardial infarction (>30 d) & $462(17.5 \%)$ & $49(35.8 \%)$ & $511(18.4 \%)$ & $<0.0001$ \\
\hline Prior percutaneous coronary intervention & $565(21.4 \%)$ & $47(34.3 \%)$ & $612(22.0 \%)$ & 0.0007 \\
\hline Prior coronary artery bypass grafting & $317(12.0 \%)$ & $52(38.0 \%)$ & $369(13.3 \%)$ & 0.0007 \\
\hline Congestive heart failure & $115(4.4 \%)$ & $28(20.4 \%)$ & $143(5.1 \%)$ & $<0.0001$ \\
\hline Early revascularization ( $\leq 90$ d post-PET) & $217(8.2 \%)$ & $18(13.1 \%)$ & $235(8.4 \%)$ & 0.0564 \\
\hline Stress protocol & & & & $<0.0001$ \\
\hline Adenosine & $205(7.8 \%)$ & $11(8.0 \%)$ & $216(7.8 \%)$ & \\
\hline Dipyridamole & $1,280(48.4 \%)$ & $96(70.1 \%)$ & $1376(49.4 \%)$ & \\
\hline Dobutamine & $115(4.4 \%)$ & $9(6.6 \%)$ & $124(4.5 \%)$ & \\
\hline Regadenoson & $1,046(39.5 \%)$ & $21(15.3 \%)$ & $1,067(38.3 \%)$ & \\
\hline \multicolumn{5}{|l|}{ Imaging parameters } \\
\hline Rest left ventricular ejection fraction (\%) & 59 [49-66] & 39 [27-54] & $58[48-66]$ & $<0.0001$ \\
\hline Stress-induced $\uparrow$ left ventricular ejection fraction & $2,060(77.9 \%)$ & $87(63.5 \%)$ & $2,147(77.2 \%)$ & 0.0002 \\
\hline Scar + ischemic myocardium (\%) & $0[0-10.3]$ & $16.2[4.4-35.3]$ & $0[0-10.3]$ & $<0.0001$ \\
\hline Ischemic myocardium (\%) & $0[0-4.4]$ & $4.4[0-10.3]$ & $0[0-5.9]$ & $<0.0001$ \\
\hline Global MFR & $1.76[1.37-2.25]$ & $1.31[1.12-1.56]$ & $1.73[1.34-2.22]$ & $<0.0001$ \\
\hline Stress global MBF (mL/min/g) & $1.82[1.25-2.54]$ & $1.26[0.92-1.82]$ & $1.80[1.23-2.52]$ & $<0.0001$ \\
\hline Rest global MBF (mL/min/g) & $1.01[0.79-1.33]$ & $1.00[0.75-1.34]$ & $1.01[0.78-1.33]$ & 0.41 \\
\hline
\end{tabular}

Numbers presented are median [interquartile range] for continuous variables and number (\%) for binary and categorical variables. 

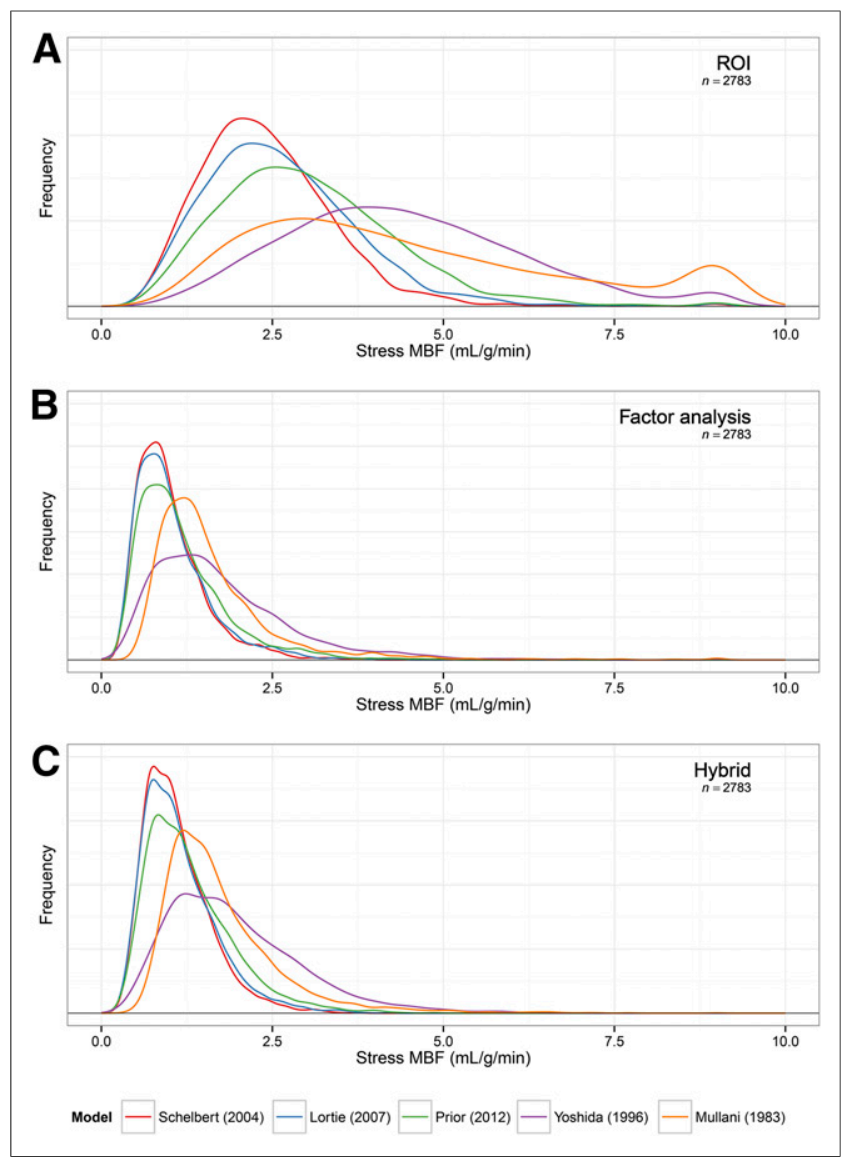

FIGURE 2. Distribution of stress MBF by input function method and ${ }^{82} \mathrm{Rb}$ extraction model. Distribution of stress MBF estimates using ROI (A), factor analysis (B), and hybrid methods (C) for estimation of input function and 5 different clinically used extraction models.

of which extraction model was used. In contrast, for each input function estimation method, all 5 extraction models generated similar distributions of MFR (Fig. 3; Supplemental Table 2). However, the distributions of MFR obtained with the ROI method were wider and are skewed toward higher values, compared with the factor analysis and hybrid methods.

Overall, when all combinations of input function estimation methods and extraction models were compared against the combination of the hybrid method with the Yoshida extraction model used in prior publications $(3,9,25-27)$, there was tremendous scatter (Supplemental Fig. 2A). Relatively little of this variability was attributable to the extraction model used (Supplemental Fig. 2B). Instead, most of the variability was attributable to differences between the input function estimation methods (Supplemental Fig. 2C). Correspondingly, correlations between stress MBF and MFR estimates were strong (Pearson $r>0.90$ ) across different extraction models, as long as the same input function estimation method was used (Supplemental Figs. 3 and 4). Importantly, correlations between measures obtained using the ROI method and the other 2 methods were only moderate (Pearson $r=0.42-0.62$ ). However, even with measures obtained using the same method for defining the input function, substantial biases in both MFR and stress MBF resulted if different extraction models were used (Supplemental Figs. 5 and 6). Finally, Cohen $\kappa$, a measure of repeatability, was used to evaluate the frequency of discrepant categorization of MFR as $<1.5,1.5-2.0$, and $>2.0$, corresponding to validated categories of clinical risk (Supplemental Fig. 7) (3,4). Agreement was generally moderate to strong between MFR measures obtained with the same input function determination method and substantially weaker between different input function methods.

\section{Prognostic Implications of Variation in Stress MBF and MFR}

We used unadjusted Poisson regression with smoothing splines to estimate the annual cardiac mortality rate as a function of stress MBF and MFR (Figs. 4 and 5). Both lower MFR and lower stress MBF were associated with a substantial increase in annualized rate of cardiac death. Clear threshold effects were seen in nearly all cases except for stress MBF computed with the ROI method. Importantly, the thresholds below which substantial increases in cardiac mortality rates were seen differed widely across different ${ }^{82} \mathrm{Rb}$ extraction models when the ROI method was used to define the input function. Somewhat less variability was seen when stress MBF measures were obtained using either the factor analysis or the hybrid methods to define the input function. Importantly, the risk versus MFR curves were remarkably similar regardless of which combination of extraction fraction model and input function determination method was used.

Last, we evaluated the incremental value of MFR and MBF measures with the continuous net reclassification improvement, comparing these measures with a base model including age, sex,

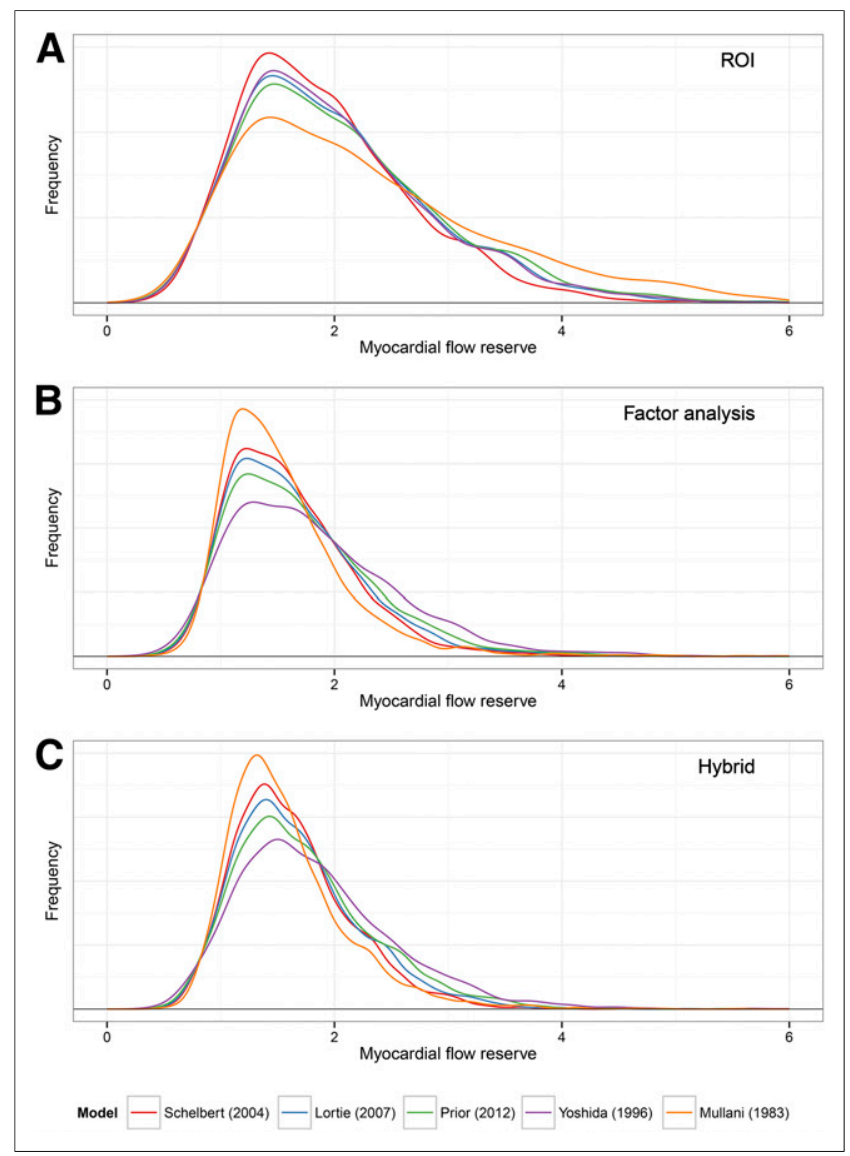

FIGURE 3. Distribution of MFR by input function method and ${ }^{82} \mathrm{Rb}$ extraction model. Distribution of MFR estimates using ROI (A), factor analysis (B), and hybrid methods (C) for estimation of input function and 5 different clinically used extraction models. 


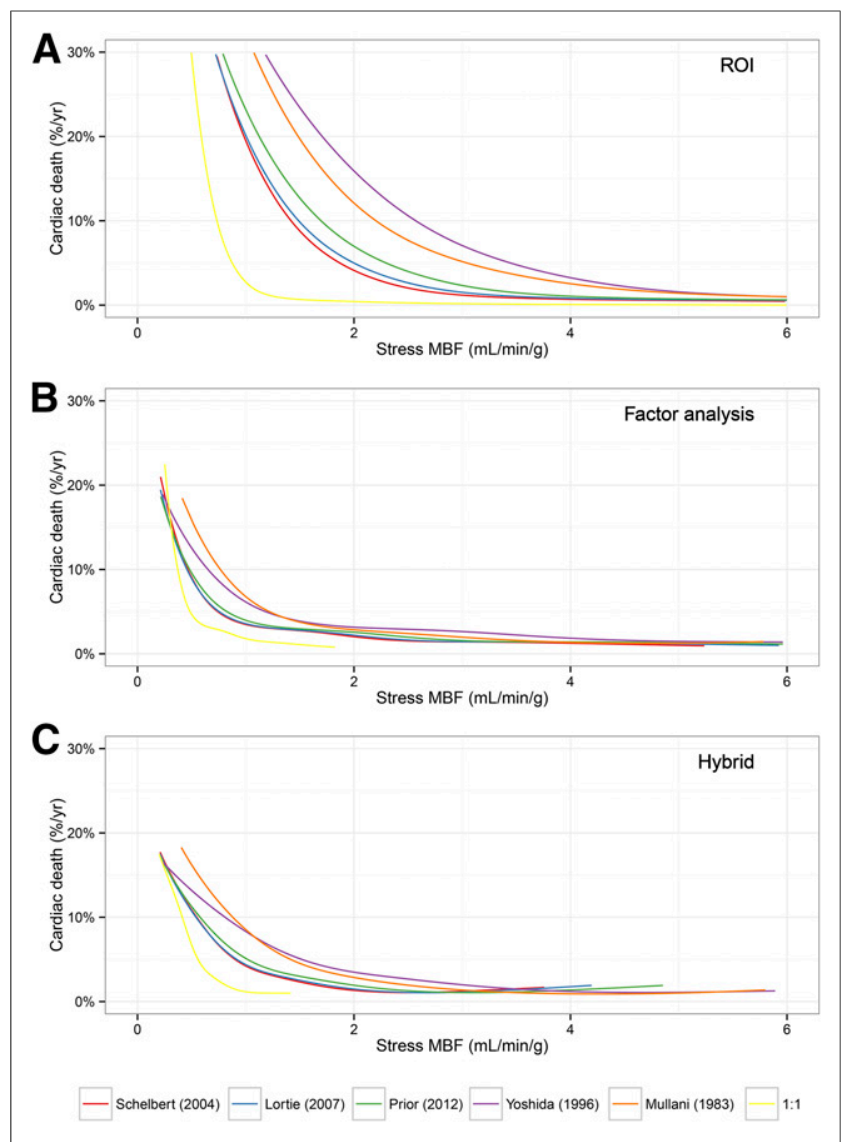

FIGURE 4. Risk of cardiac death versus stress MBF. Annual rate of death from cardiac causes as function of stress MBF computed using ROI (A), factor analysis (B), and hybrid methods (C) for estimation of input function and 5 different clinically used extraction models for ${ }^{82} \mathrm{Rb}$ and 1 counterfactual model assuming 100\% extraction (1:1). Curves were generated using Poisson regression with smoothing splines.

hypertension, dyslipidemia, diabetes, smoking, family history of $\mathrm{CAD}$, prior $\mathrm{CAD}$, body mass index, chest pain, dyspnea, early revascularization ( $<90 \mathrm{~d})$, left ventricular ejection fraction, left ventricular ejection fraction reserve, and the combined extent and severity of scar and ischemia (Supplemental Fig. 8A; Supplemental Table 3). We found that NRI was similar when the ROI or hybrid methods were used with any of the extraction models. NRIs for MFR computed using factor analysis were marginally smaller than NRIs from ROI or hybrid methods, though with substantial overlap of confidence intervals. Importantly, the NRIs for stress MBF measures were all lower than for MFR and were highly inconsistent depending on which method was used to define the input function (Supplemental Fig. 8B). Risk discrimination, as assessed by the c-index, was comparable for all combinations of input function estimation method and extraction model for both stress MBF and MFR (Supplemental Fig. 9).

\section{DISCUSSION}

We have demonstrated in a large clinical cohort that the choice of method for determination of input function and model for correction of the nonlinear extraction of ${ }^{82} \mathrm{Rb}$ has a substantial impact on the resulting MBF and MFR measurements. Stress MBF measurements are highly variable, depending on technique used, especially when the ROI method is used to generate the input function. Indeed, when used with the ROI method, the extraction models of Mullani et al. (11) and Yoshida et al. (13) lead to extremely high stress MBF estimates, likely beyond what would be expected in clinical cohorts. In contrast, estimates of MFR are more similar, although substantial differences can still exist when different input function methods are used. Importantly, these sources of variability have a substantial impact on the relationship between specific values of stress MBF or MFR and the rate of cardiac mortality as well as on incremental risk reclassification. Overall, we found that MFR measures were substantially more consistent across variations in input function method and extraction model than stress MBF measures.

These data are inconsistent with prior, smaller studies (19-21), suggesting that measures with different software packages, some of which implement different choices with regard to the method of determination of the input function and the extraction model for ${ }^{82} \mathrm{Rb}$, may not be interchangeable clinically. Potential reasons for this could include limited power in prior studies, which had cohort sizes of 25-90 as compared with the 2,783 subjects analyzed for this study. Second, the other factors may have offset some of the differences. For example, temporal duration of frames used in dynamic acquisitions $(28,29)$, filtering (30), and reconstruction algorithms $(31,32)$ may also affect measured MBFs. Finally, subtle differences between the software method used for defining the ROI and the methods used in prior studies may also contribute.

Importantly, both $K_{1}$ and $k_{2}$ were substantially affected by the method used to estimate the input function. Although $K_{1}$ is directly related to $\mathrm{MBF}, k_{2}$ is related to retention and washout of the tracer from tissue. The $K_{1}$ measurements from the hybrid and factor analysis methods are lower, in general, than those from the ROI method (Supplemental Fig. 1). In this range of $K_{1}$ values, the differences between the various extraction models for ${ }^{82} \mathrm{Rb}$ are modest (Fig. 1). In contrast, the higher $K_{1}$ values determined by the ROI method lead to greater divergences in estimated blood flow for the various ${ }^{82} \mathrm{Rb}$ extraction models. It is possible that the larger variability in $K_{1}$ and stress MBF with the ROI method could potentially improve sensitivity or specificity, although our analysis did not evaluate the use of these measures for diagnostic purposes.

Prior studies have demonstrated increased washout of ${ }^{82} \mathrm{Rb}$ from nonviable myocardium $(33,34)$. The application of this concept relies on accurate quantification of $k_{2}$, which we have demonstrated varies markedly based on whether factor analysis, hybrid, or ROI methods are used. Importantly, $K_{1}$ and $k_{2}$ are substantially correlated when the ROI method is used and not meaningfully correlated for the hybrid and factor analysis methods. The observed correlation between $K_{1}$ and $k_{2}$ with the ROI method has no obvious physiologic basis and suggests that the ROI method may deliver estimates for these parameters with greater bias than factor analysis, as suggested by prior simulation studies (17).

These results have several important implications for clinical risk assessment. First, MFR estimates appear to be substantially more consistent than stress MBF measures. The potential cut points of stress MBF to define low-, intermediate-, and high-risk groups vary considerably depending on the method of input function determination and the extraction model used, limiting widespread clinical translation in the absence of much greater standardization than exists today. In contrast, MFR measures deliver more consistency in the relationship between the degree of reduction in MFR and the absolute cardiac mortality rate. In particular, an MFR greater than 2.0 is associated with favorable prognosis across all of the variations we evaluated and is 


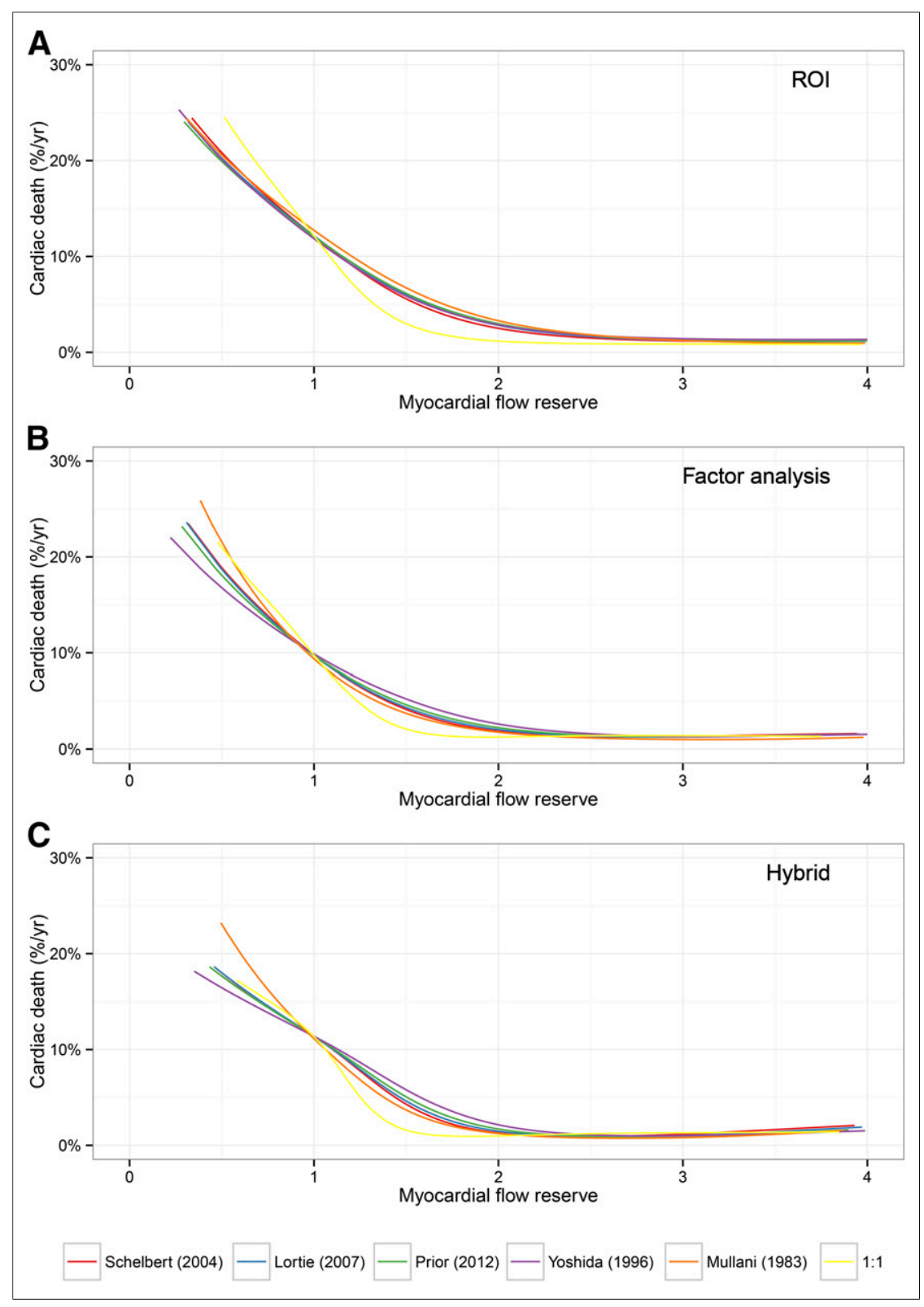

FIGURE 5. Risk of cardiac death versus MFR. Annual rate of death from cardiac causes as function of MFR computed using ROI (A), factor analysis (B), and hybrid methods (C) for estimation of input function and 5 different clinically used extraction models for ${ }^{82} \mathrm{Rb}$ and 1 counterfactual model assuming 100\% extraction (1:1). Curves were generated using Poisson regression with smoothing splines.

consistent with the results seen in multiple independent clinical cohorts (1-6). Consequently, these data support the use of both rest and stress imaging to enable estimation of MFR rather than stress-only imaging for which only stress MBF could be measured. Although this combination of both rest and stress imaging results in increased exposures to patients, the effective dose remains modest, compared with SPECT radiopharmaceuticals.

Second, application of the large body of prognostic and diagnostic data for ${ }^{82} \mathrm{Rb}$ PET to other perfusion tracers is uncertain and carries several important concerns. The same method for determination of the input function would be required for the other tracers, particularly for comparison of stress MBF. Translation of cut points across tracers would require highly re- liable extraction models for both tracers. Unfortunately, for ${ }^{82} \mathrm{Rb}$ substantial controversy exists as to the correct extraction model. All current extraction models incorporate biases and limitations related to the instrumentation, methodology, and reference standard used for their derivation and are thus more accurately thought of as calibration functions. MFR measures are potentially more reproducible across tracers than stress MBF metrics, although this has yet to be tested rigorously.

Important limitations of our work include that we only corrected for spillover of right ventricular blood pool with the hybrid and factor analysis methods and not with the ROI method. The impact of this discrepancy was minor (supplemental results). Furthermore, MFR and stress MBF were modeled as linear predictors for the purposes of NRI and c-index computation. Because we have demonstrated that risk of cardiac mortality is related to stress MBF and MFR in a highly nonlinear manner, our analyses of NRI and c-indices may substantially underestimate the potential incremental value of stress MBF and MFR. Finally, the cause of death was unknown in a few cases. This uncertainty combined with potential inaccuracies in death certificates could result in modest under- or overestimation of actual mortality rates.

\section{CONCLUSION}

Variability in the technique used to determine the input function and the extraction model for ${ }^{82} \mathrm{Rb}$ can lead to large differences in estimates for stress MBF and, to a lesser extent, MFR. The greater variability in stress MBF measures results in substantial variation in the relationship between stress MBF and annual cardiac mortality, depending on the technique used. In contrast, MFR is considerably more consistent, with similar relationships between MFR and cardiac mortality, regardless of technique used. These results suggest that without careful standardization of methods, stress-only imaging cannot be readily generalized across multiple sites for clinical applications and in multicenter research studies.

\section{DISCLOSURE}

The costs of publication of this article were defrayed in part by the payment of page charges. Therefore, and solely to indicate this fact, this article is hereby marked "advertisement" in accordance with 18 USC section 1734 . This work was supported in part by National Institutes of Health grant T32HL094301-02, the Blahd Research grant from the Society of Nuclear Medicine and Molecular Imaging, and research grants from the Intersocietal Accreditation 
Council and INVIA Medical Imaging Solutions. Dr. Ficaro owns equity in INVIA Medical Imaging Solutions. Dr. Sitek receives royalties related to factor analysis. Drs. Lee and Moody are employed by INVIA Medical Imaging Solutions.

\section{REFERENCES}

1. Herzog BA, Husmann L, Valenta I, et al. Long-term prognostic value of ${ }^{13} \mathrm{~N}$ ammonia myocardial perfusion positron emission tomography: added value of coronary flow reserve. J Am Coll Cardiol. 2009;54:150-156.

2. Tio RA, Dabeshlim A, Siebelink H-MJ, et al. Comparison between the prognostic value of left ventricular function and myocardial perfusion reserve in patients with ischemic heart disease. J Nucl Med. 2009;50:214-219.

3. Murthy VL, Naya M, Foster CR, et al. Improved cardiac risk assessment with noninvasive measures of coronary flow reserve. Circulation. 2011;124: 2215-2224

4. Ziadi MC, Dekemp RA, Williams KA, et al. Impaired myocardial flow reserve on rubidium-82 positron emission tomography imaging predicts adverse outcomes in patients assessed for myocardial ischemia. J Am Coll Cardiol. 2011;58:740-748.

5. Fukushima K, Javadi MS, Higuchi T, et al. Prediction of short-term cardiovascular events using quantification of global myocardial flow reserve in patients referred for clinical ${ }^{82} \mathrm{Rb}$ PET perfusion imaging. J Nucl Med. 2011;52:726-732.

6. Farhad H, Dunet V, Bachelard K, Allenbach G, Kaufmann PA, Prior JO. Added prognostic value of myocardial blood flow quantitation in rubidium82 positron emission tomography imaging. Eur Heart J Cardiovasc Imaging. 2013;14:1203-1210.

7. Ziadi MC, Dekemp RA, Williams K, et al. Does quantification of myocardial flow reserve using rubidium- 82 positron emission tomography facilitate detection of multivessel coronary artery disease? J Nucl Cardiol. 2012;19:670-680.

8. Fiechter M, Ghadri JR, Gebhard C, et al. Diagnostic value of ${ }^{13} \mathrm{~N}$-ammonia myocardial perfusion PET: added value of myocardial flow reserve. J Nucl Med. 2012;53:1230-1234.

9. Naya M, Murthy VL, Taqueti VR, et al. Preserved coronary flow reserve effectively excludes high-risk coronary artery disease on angiography. $\mathrm{J} \mathrm{Nucl} \mathrm{Med}$. 2014;55:248-255.

10. Joutsiniemi E, Saraste A, Pietilä M, et al. Absolute flow or myocardial flow reserve for the detection of significant coronary artery disease? Eur Heart $J$ Cardiovasc Imaging. 2014;15:659-665.

11. Mullani NA, Goldstein RA, Gould KL, et al. Myocardial perfusion with rubidium-82. I. Measurement of extraction fraction and flow with external detectors. J Nucl Med. 1983;24:898-906.

12. Glatting G, Bergmann KP, Stollfuß JC, et al. 1011-116 Myocardial Rb extraction fraction: determination in humans. J Am Coll Cardiol. 1995;25:364A-365A.

13. Yoshida K, Mullani N, Gould KL. Coronary flow and flow reserve by PET simplified for clinical applications using rubidium-82 or nitrogen-13-ammonia. J Nucl Med. 1996;37:1701-1712.

14. Schelbert HR. Positron emission tomography of the heart: methodology, findings in the normal and the diseased heart, and clinical applications. In: PET: Molecular Imaging and Its Biological Applications. New York, NY: Springer; 2004:389-508.

15. Lortie M, Beanlands RSB, Yoshinaga K, Klein R, DaSilva JN, deKemp RA. Quantification of myocardial blood flow with ${ }^{82} \mathrm{Rb}$ dynamic PET imaging. Eur J Nucl Med Mol Imaging. 2007;34:1765-1774.
16. Prior JO, Allenbach G, Valenta I, et al. Quantification of myocardial blood flow with ${ }^{82} \mathrm{Rb}$ positron emission tomography: clinical validation with ${ }^{15} \mathrm{O}$-water. Eur J Nucl Med Mol Imaging. 2012;39:1037-1047.

17. El Fakhri G, Sitek A, Guérin B, Kijewski MF, Di Carli MF, Moore SC. Quantitative dynamic cardiac ${ }^{82} \mathrm{Rb}$ PET using generalized factor and compartment analyses. J Nucl Med. 2005;46:1264-1271.

18. El Fakhri G, Kardan A, Sitek A, et al. Reproducibility and accuracy of quantitative myocardial blood flow assessment with ${ }^{82} \mathrm{Rb}$ PET: comparison with ${ }^{13} \mathrm{~N}$ ammonia PET. J Nucl Med. 2009;50:1062-1071.

19. Dekemp RA, Declerck J, Klein R, et al. Multisoftware reproducibility study of stress and rest myocardial blood flow assessed with 3D dynamic PET/CT and a 1-tissuecompartment model of ${ }^{82} \mathrm{Rb}$ kinetics. J Nucl Med. 2013;54:571-577.

20. Nesterov SV, Deshayes E, Sciagrà R, et al. Quantification of myocardial blood flow in absolute terms using ${ }^{82} \mathrm{Rb}$ PET imaging: Results of RUBY-10 Study. JACC Cardiovasc Imaging. October 1, 2014 [Epub ahead of print].

21. Tahari AK, Lee A, Rajaram M, et al. Absolute myocardial flow quantification with ${ }^{82} \mathrm{Rb}$ PET/CT: comparison of different software packages and methods. Eur J Nucl Med Mol Imaging. 2014;41:126-135.

22. Cerqueira MD, Weissman NJ, Dilsizian V, et al. Standardized myocardial segmentation and nomenclature for tomographic imaging of the heart: a statement for healthcare professionals from the Cardiac Imaging Committee of the Council on Clinical Cardiology of the American Heart Association. Circulation. 2002; 105:539-542.

23. Crone $\mathrm{C}$. The permeability of capillaries in various organs as determined by use of the "indicator diffusion" method. Acta Physiol Scand. 1963;58:292-305.

24. Renkin EM. Transport of potassium-42 from blood to tissue in isolated mammalian skeletal muscles. Am J Physiol. 1959;197:1205-1210.

25. Murthy VL, Naya M, Foster CR, et al. Association between coronary vascular dysfunction and cardiac mortality in patients with and without diabetes mellitus. Circulation. 2012;126:1858-1868.

26. Murthy VL, Naya M, Foster CR, et al. Coronary vascular dysfunction and prognosis in patients with chronic kidney disease. JACC Cardiovasc Imaging. 2012;5:1025-1034.

27. Murthy VL, Naya M, Taqueti VR, et al. Effects of sex on coronary microvascular dysfunction and cardiac outcomes. Circulation. 2014;129:2518-2527.

28. Kolthammer JA, Muzic RF. Optimized dynamic framing for PET-based myocardial blood flow estimation. Phys Med Biol. 2013;58:5783-5801.

29. Lee B, Moody J, Murthy V, Corbett J, Ficaro E. Effects of temporal sampling on PET myocardial blood flow estimates [abstract]. J Nucl Med. 2014;55 (suppl 1):1775.

30. Lee B, Moody J, Sitek A, et al. Effects of filtering on Rb-82 myocardial blood flow estimates [abstract]. J Nucl Med. 2013;54(suppl 2):1659.

31. Armstrong IS, Tonge CM, Arumugam P. Impact of point spread function modeling and time-of-flight on myocardial blood flow and myocardial flow reserve measurements for rubidium-82 cardiac PET. J Nucl Cardiol. 2014;21:467-474.

32. Chen GP, Branch KR, Alessio AM, et al. Effect of reconstruction algorithms on myocardial blood flow measurement with ${ }^{13} \mathrm{~N}$-ammonia PET. J Nucl Med. 2007;48:1259-1265.

33. Gould KL, Yoshida K, Hess MJ, Haynie M, Mullani N, Smalling RW. Myocardial metabolism of fluorodeoxyglucose compared with cell membrane integrity for the potassium analogue rubidium- 82 for assessing infarct size in man by PET. $J$ Nucl Med. 1991;32:1-9.

34. vom Dahl J, Muzik O, Wolfe ER, Allman C, Hutchins G, Schwaiger M. Myocardial rubidium-82 tissue kinetics assessed by dynamic positron emission tomography as a marker of myocardial cell membrane integrity and viability. Circulation. 1996;93:238-245. 